\title{
Perturbation of zero surfaces
}

\author{
Alexander G. Ramm* \\ Department of Mathematics, Kansas State University, Manhattan, USA
}

\section{Abstract}

It is proved that if a smooth function $u(x), x \in \square^{3}$, such that $\inf _{s \in S}\left|u_{N}(s)\right|>0$, where $u_{N}$ is the normal derivative of $\mathrm{u}$ on $\mathrm{S}$, has a closed smooth surface $\mathrm{S}$ of zeros, then the function $u(x)+\in v(x)$ has also a closed smooth surface $S_{\in}$ of zeros. Here $v$ is a smooth function and $\in>0$ is a sufficiently small number.

\section{Introduction}

Let $D \subset \square^{3}$ be a bounded domain containing inside a connected closed $C^{3}$-smooth surface $S$, which is the set of zeros of a function $\left.u \in C^{3} D\right)$, so that Consider the scattering problem:

$$
u \mid s=0
$$

Let $N=N_{s}$ be the unit normal to $S$, such that $u_{N}=|\nabla u(s)|$, where $u_{N}$ is the normal derivative of $u$ on $S$. Let $u_{\epsilon}:=u+\in v$, where $v \in C^{3}(D)$ and $\in>0$ is sufficiently small. Assume that

$$
\inf _{s \in S}|\nabla u(s)| \geq 2 c_{1}>0, c_{1}=\text { const }>0 .
$$

The purpose of this paper is to prove Theorem 1.

Theorem 1. Under the above assumptions there exists a smooth closed surface $S_{\in}$ such that $u_{\epsilon}=0$ on $S_{\epsilon}$.

In Section 2 Theorem 1 is proved.

Although there are many various results on perturbation theory, see [2], [3], the result formulated in Theorem 1 is new.

\section{Proof of Theorem 1}

Consider the following equation for $t$ :

$$
u(s+t N+\in v s+t N=0
$$

where $N=N(s$ is the normal to $S$ at the point $s$ and $t$ is a parameter. Using the Taylor's formula and relation (1), one gets from (3)

$$
t \nabla u\left(s \cdot N+\in \nabla v s \cdot N+\in v s+t^{2} \phi=0,\right.
$$

where $t^{2} \phi$ is the Lagrange remainder in the Taylor's formula and

$$
\phi=\sum_{i, j=1}^{3}\left[u_{x_{i} x_{j}}\left(s+\theta t N+\in v_{x_{i} x_{j}} s+\theta t N\right] N_{i} N_{j}, \theta \in(0,1\right.
$$

Since the functions $u$ and $v$ belong to $C^{3}(D)$, the function $\phi=\phi(t, s, \in$ has a bounded derivative with respect to $t$ uniformly with respect to $s \in S$ and $\in \in(0,1$.

Consider equation (4) as an equation for $t=t(s)$ in the space $C(S)$. Rewrite (4) as

$$
t=-\in \nabla u\left(s \cdot N+\in \nabla v s \cdot N^{-1} v s-t^{2} \phi \nabla u s \cdot N+\in \nabla v s \cdot N^{-1}:=B t .(6)\right.
$$

Let us check that the operator B satisfies the contraction mapping theorem in the set
$M:=t: \max _{s \in S} \mid t\left(s-\in \nabla u s \cdot N+\in \nabla v s \cdot N^{-1} v s \mid \leq \delta\right.$,

where $\delta>0$ is a small number, and $M \in C(S$.

First, one should check that $B$ maps $M$ into itself. One has

$\left.\max _{s \in S} \mid B t s\right)-\in(\nabla u(s) \cdot N+\in \nabla v(s) \cdot N)^{-1} v(s) \mid \leq \max _{s \in S} \frac{t^{2}|\phi|}{\nabla u(s) \cdot N+\in \nabla v(s) \cdot N}$.

We have chosen $N$ so that $\nabla u(s) \cdot N=|\nabla u(s)|$. This is possible because equation (1) implies that $\nabla u s)$ is orthogonal to $S$ at the point $s \in S$. Assumption (2) implies that for sufficiently small $\in$ one has

$\inf _{s \in S}\left|\nabla u_{\in}(s)\right| \geq c_{1}$.

Since $\phi$ is continuously differentiable, one has

$$
\sup _{s \in S, t \in(0,1)}|\phi(t, s, \in)| \leq c_{2} \text {. }
$$

Therefore, if

$\mid t \quad s) \mid \leq \delta$,

Then

$\frac{t^{2}(s)|\phi(t, s, \epsilon)|}{|\nabla u(s)|+\in \nabla v(s) \cdot N} \leq \frac{c_{2}}{c_{1}} \leq \delta$,

Provided that

$\frac{c_{2}}{c_{1}} \delta \leq 1$.

Thus, if (13) holds then B maps M into itself.

Let us check that $\mathrm{B}$ is a contraction mapping on $\mathrm{M}$. One has

$\left|B t_{1}-B t_{2}\right| \leq c_{1}^{-1}\left|t_{1}^{2} \phi\left(t_{1}, s, \in\right)-t_{2}^{2} \phi\left(t_{2}, s, \in\right)\right| \leq c_{3}\left|t_{1}-t_{2}\right|$

Where $\left.c_{3} \in 0,1\right)$ if $\delta$ is sufficiently small. Indeed,

Correspondence to: Alexander G. Ramm, Department of Mathematics, Kansas State University, Manhattan, KS 66506, USA, E-mail: ramm@math.ksu.edu

Key words: zero surfaces, perturbation theory

Received: October 28, 2016; Accepted: December 24, 2016; Published: December 27, 2016 


$$
c_{3}=\max _{s \in S, t \leq \delta}\left(2 t|\phi(t, s, \in)|+t^{2}\left|\frac{\partial \phi}{\partial t}\right|\right) \leq c_{4} \delta<1,
$$

if $\delta$ is suciently small. Here $c_{4}$ is a constant.

Thus, $B$ is a contraction on $M$. By the contraction mapping principle, equation (6) is uniquely solvable for $t$. Its solution $t=t(s)$ allows one to construct the zero surface $S_{\epsilon}$ of the function $u_{\in}$ by the equation $r=s+t(s) N$, where $r=r(s)$ is the radius vector of the points on $S_{\epsilon}$.

Theorem 1 is proved.

Remark 1. Condition (2) is a sufficient condition for the validity of Theorem 1. Although this condition is not necessary, if it does not hold one can construct counterexamples to the conclusion of Theorem 1. For example, assume that $u(x) \geq 0$ and $u(x)=0$ on S, and let $v>0$ and $\in>0$. Then the function $u_{\epsilon}=u+\in v$ does not have zeros in $\square^{3}$.

Remark 2. In scattering theory the following question is of interest: assume that $u(x)$ is an entire function of exponential type, $u(x)=\int_{\mathcal{s}^{2}} e^{i k \beta \cdot x} f(\beta) d \beta$, where $f \in L^{2}\left(S^{2}\right), S^{2}$ is the unit sphere in $\square^{3}$. Assume that $u=0$ on $\mathrm{S}$, where $S$ is a closed smooth connected surface in $\square^{3}$.

Is there another closed smooth connected surface of zeros of an entire function $u_{\in}$ of exponential type, $u_{\epsilon}=\int_{s^{2}} e^{i k \beta \cdot x}[f(\beta)+\in g(\beta)] d \beta$, where $g \in L^{2}\left(S^{2}\right)$ and $\in>0$ is a small parameter?
We will not use Theorem 1 since assumption (2) may not hold, but sketch an argument, based on the fact that $S$ in the above question is the intersection of an analytic set with $\square^{3}$, see, for example, [1] for the definition and properties of analytic sets. The functions $u$ and $u_{\epsilon}$ in Remark 2 solve the differential equation

$$
\nabla^{2} u+k^{2} u=0 \text { in } \square^{3}, k^{2}=\text { const }>0 .
$$

The function $u_{N}$ may vanish on $S$ at most on the closed set $\sigma \subset S$ which is of the surface measure zero (by the uniqueness of the solution to the Cauchy problem for equation (16)). For every point $s \in S \backslash \sigma$ the argument given in the proof of Theorem 1 yields the existence of $t(s)$, the unique solution to (6). Since $S$ is real a--lytic the set $S_{\epsilon}$, defined in the proof of Theorem 1, is analytic and is a part of the analytic set defined by the equation $u_{\epsilon}=0$. In our problem $S$ is a bounded closed real analytic surface. The set $S_{\in}$ can be continued analytically to an analytic set which intersects the real space $\square^{3}$ over a real analytic surface $S_{\epsilon}$. It is still an open problem to prove (or disprove) that the analytic continuation of the set $S_{\epsilon}$ intersects $\square^{3}$ over a bounded closed real analytic surface $S_{\in} \in \square^{3}$.

\section{References}

1. Fuks B (1963) Theory of analytic functions of several complex variables, AMS, Providence RI.

2. Kato T (1984) Perturbation theory for linear operators, Springer Verlag, New York.

3. Ramm AG (2005) Inverse problems, Springer, New York.

Copyright: (C2016 Ramm AG. This is an open-access article distributed under the terms of the Creative Commons Attribution License, which permits unrestricted use, distribution, and reproduction in any medium, provided the original author and source are credited. 